\title{
有机质孔隙对页岩气流动能力影响研究
}

\author{
1. 中国石油大学(华东)石油工程学院, 青岛 266580; \\ 2. 中国石化油田勘探开发事业部, 北京 100029 \\ *联系人, E-mail: RCOGFR_UPC@126.com
}

姚军 ${ }^{*}$, 宋文辉 ${ }^{1}$, 李阳 ${ }^{2}$, 孙海 $^{1}$, 杨永飞 ${ }^{1}$, 张䂞 $^{1}$

收稿日期: 2017-02-20; 接受日期: 2017-05-26; 网络出版日期: 2017-08-13

国家自然科学基金(编号: 51490654, 51234007, 51504276, 51504277)、高等学校学科创新引智计划(编号: B08028)、山东省自然科学基金 (编号: ZR2014EEP018, ZR2014EL016, ZR2014EL017)、国家油气重大专项(编号: 2016ZX05061)和中国石油大学(华东)研究生创新工程(编 号：YCX2017019)资助项目

\begin{abstract}
摘要＼cjkstart页岩中存在有机质孔隙和无机质孔隙, 目前页岩气表观渗透率模型忽略了孔隙类型、孔隙半径分布 对气体流动能力的影响. 本文基于页岩二维剖光扫描电镜图像数值重建页岩三维数字岩心, 并提取孔隙相中轴 拓扑结构.根据地质上对有机质孔隙空间分布、有机质孔隙和无机质孔隙半径分布的认识, 在对应孔隙喉道位 置, 充填满足对应孔隙半径分布以及空间分布关系的有机质孔隙和无机质孔隙, 建立3种不同有机质孔隙分布 模式的页岩孔隙网络模型: 有机质孔隙条带状分布、有机质孔隙连片分布、有机质孔隙分散在无机质孔隙中. 有机质孔隙中气体流动考虑吸附解吸、表面扩散、黏性流、努森扩散, 无机质孔隙中气体流动考虑黏性流、 努森扩散, 气体性质计算考虑真实气体效应以及在微纳米孔道中相态变化. 结果表明, 当有机质孔隙半径分布 小于无机质孔隙半径分布, 有机质孔隙成条带状分布模型气体流动能力最强, 有机质孔隙连片分布模型气体流 动能力最弱. 当有机质孔隙半径分布大于无机质孔隙半径分布, 有机质孔隙成条带状分布模型气体流动能力最 强, 有机质孔隙分散在无机质孔隙模型气体流动能力最弱. 本文的研究对认识页岩气流动能力具有显著意义.
\end{abstract}

关键词页岩气, 运移机制, 孔隙网络模型, 有机质孔隙, 无机质孔隙

PACS: $47.56 .+\mathrm{r}, 51.10 .+\mathrm{y}, 91.60 . \mathrm{Np}$

页岩微纳米孔道中气体流动能力是目前非常规领 域研究热点 ${ }^{[1]}$. 吸附气和自由气共存于页岩孔隙中. 气 体在页岩中的质量传输有以下 3 种机制 ${ }^{[2-4]}$ : 黏性流、 Knudsen扩散、表面扩散. 如图1所示, 同种气体分子 与分子之间碰撞产生黏性流; 分子与壁面碰撞产生 Knudsen扩散; 吸附在孔隙壁面的气体分子沿孔隙表 面蠕动产生表面扩散. 由于连续性假设在微纳米孔道
中不适用, 一般采用气体分子运动自由程与孔隙半径 的比值Knudsen数判定页岩中的气体运移模式 ${ }^{[5]}$ (图2), 不同Knudsen数对应气体运移机制不同. Beskok等人 ${ }^{[6]}$ 和Civan等人 ${ }^{[7-9]}$ 建立了基于Knudsen数的气体表观渗 透率模型, Javadpour课题组 ${ }^{[5,10-12]}$ 和 $\mathrm{Wu}$ 等人 ${ }^{[13-15]}$ 建立 了基于多重气体运移机制叠加的气体表观渗透率模 型. 但目前基于单管分析的页岩气表观渗透率模型忽

引用格式: 姚军, 宋文辉, 李阳, 等. 有机质孔隙对页岩气流动能力影响研究. 中国科学: 物理学 力学 天文学, 2017, 47: 094702

Yao J, Song W H, Li Y, et al. Study on the influence of organic pores on shale gas flow ability (in Chinese). Sci Sin-Phys Mech Astron, 2017, 47: 094702, doi: 10.1360/SSPMA2017-00040 


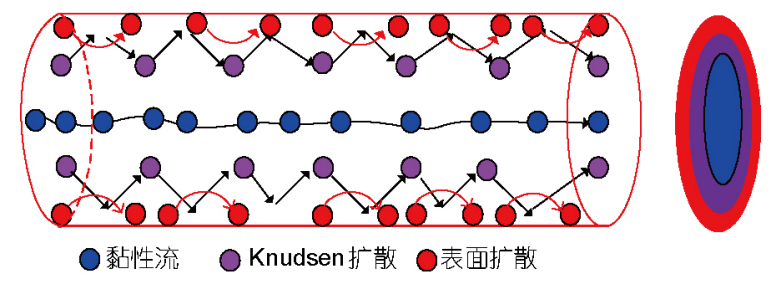

图 1 (网络版彩图)气体传输机制

Figure 1 (Color online) Gas transport mechanisms in porous media.

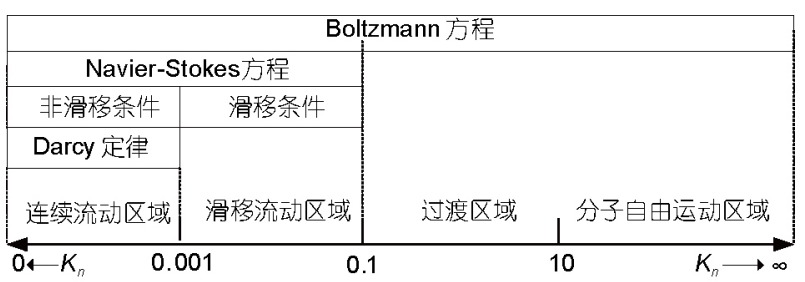

图 2 基于Knudsen数多孔介质流动形态和控制方程的分类 Figure 2 Classification of the gas flow regimes and governing equations based on Knudsen number.

略了页岩地层的非均质性, 并且没有考虑有机质孔隙 与无机质孔隙在气体传输、空间分布、孔隙半径分 布上的差异. 此外, 目前研究页岩气体流动并没有考 虑气体在微纳米孔道中的相态变化.

Fishman等人 ${ }^{[16,17]}$ 通过分析有机质孔隙和无机质 孔隙结构, 认为有机质孔隙和无机质孔隙半径通常在 $100 \mathrm{~nm}$ 以内. Mastalerz等人 ${ }^{[18]}$ 研究认为有机质孔隙半 径范围在5-70 nm. 虽然常规认识认为有机质孔隙半 径小于无机质孔隙半径, 但也存在有机质孔隙半径大 于无机质孔隙半径的情况 ${ }^{[19]}$. Loucks等人 ${ }^{[20]}$ 认为有机 质孔隙平均半径为 $100 \mathrm{~nm}$, 并给出了 3 种有机质分布 模式(图3). 图3中A模式有机质孔隙成条带状分布, 无 机质孔隙介于有机质孔隙之间, B模式有机质孔隙连 片分布, 交错分布于无机质孔隙中, C模式有机质孔隙 零散分布在无机质孔隙中.

目前普遍认为无机质孔隙如黏土孔隙具有大量吸 附气是建立在固-气界面吸附理论基础上, 其对页岩气 藏不适应 ${ }^{[21]}$. 由于黏土矿物具有极强的亲水性, 实际 储层黏土孔隙表面吸附水膜, 并且孔隙中存在一定毛 细水及可动水 ${ }^{[22-26]}$. 在黏土孔隙表面存在水膜情况下, 大多数甲烷分子表现为聚集在孔隙中心, 或者吸附在 水膜上, 而并非吸附在黏土表面 ${ }^{[27,28]}$, 这就在很大程度 上降低了黏土对甲烷的吸附能力. 相比于有机质对甲 烷的强吸附能力, 无机质孔隙吸附能力可以忽略 ${ }^{[21]}$.

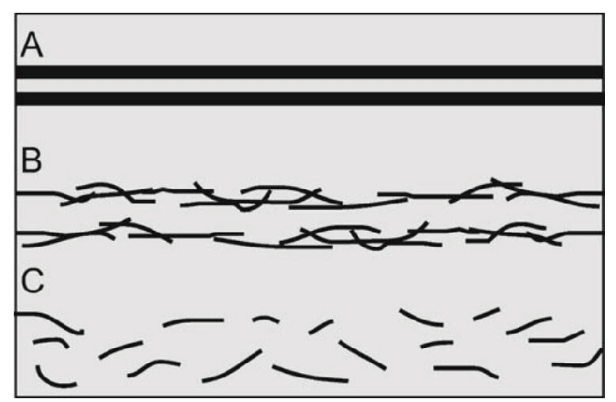

图 3 有机质分布模式 ${ }^{[20]}$

Figure 3 Organic matter distribution pattern [20].

Wang等人 ${ }^{[29]}$ 通过氮气和二氧化碳页岩吸附实验发现 气体主要吸附在有机质微孔隙中, Heller等人 ${ }^{[30]}$ 的吸附 实验结果发现活性炭最大甲烷吸附能力 $\left(5 \times 10^{3} \mathrm{scf} / \mathrm{ton}\right)$ 远大于伊利石最大甲烷吸附能力 (100 scf/ton) 和高岭 石最大甲烷吸附能力 (20 scf/ton). 在模拟页岩气流 动和气藏储量计算中一般忽略无机质孔隙中的吸附 气 $^{[31,32]}$.

本文首先建立了有机质孔隙无机质孔隙气体传 导率方程, 然后对两种不同类型孔隙单管流动能力进 行了分析. 有机质孔隙中气体流动考虑吸附解吸、表 面扩散、黏性流、努森扩散, 无机质孔隙中气体流动 考虑黏性流、努森扩散.气体性质计算考虑真实气体 效应以及气体在微纳米孔道产生的相态变化. 由此基 于图3中的有机质分布模式, 采用马尔科夫链-蒙特卡 洛(Markov chain Monte Carlo, MCMC)方法基于页岩二 维剖光扫描电镜图像重建页岩三维数字岩心, 并提取 孔隙相中轴拓扑结构, 在对应孔隙喉道, 充填满足对 应孔隙半径分布以及空间分布关系的有机质孔隙和 无机质孔隙, 建立了 3 种页岩孔隙网络模型: 有机质孔 隙成条带状分布的 $\mathrm{A}$ 模式页岩孔隙网络模型、有机质 孔隙连片分布的B模式页岩孔隙网络模型、有机质孔 隙分散在无机质孔隙中的 $C$ 模式页岩孔隙网络模型. 基于 3 种页岩孔隙网络模型, 讨论了不同有机质孔隙 半径分布下气体流动能力的差异.

\section{1 气体传导率方程}

\section{1 吸附解吸对流动半径的影响}

页岩中吸附气量可采用Langmuir等温吸附表示, 吸附气在孔隙壁面的覆盖度可表示为 
$\theta=\frac{p_{o} / Z}{p_{\mathrm{L}}+p_{o} / Z}$

考虑吸附解吸后有机质孔隙中自由气流动半径 可表示为

$r_{\text {eff_or }}=r-d_{m} \theta$.

\section{2 真实气体效应和相态变化对气体性质的影响}

采用Islam等人 ${ }^{[33]}$ 提出的修正格式的范德华方程 描述微纳米孔道气体临界压力临界温度变化

$T_{c}=\frac{8}{27 b R}\left[a-2 \sigma^{3} \varepsilon N^{2} \frac{d_{m}}{r_{\text {eff }}}\left(2.6275-0.6743 \frac{d_{m}}{r_{\text {eff }}}\right)\right]$,

$P_{c}=\frac{8}{27 b^{2}}\left[a-2 \sigma^{3} \varepsilon N^{2} \frac{d_{m}}{r_{\text {eff }}}\left(2.6275-0.6743 \frac{d_{m}}{r_{\text {eff }}}\right)\right]$,

其中, 有效半径 $r_{\mathrm{eff}}$ 在无机质孔隙中为孔隙半径 $r$, 有机 质孔隙中为自由气流动半径 $r_{\text {eff_or }}$.

气体压缩因子 $Z$ 采用如下公式进行计算 ${ }^{[34-36]}$ :

$P_{\mathrm{pr}}=\frac{p_{o}}{P_{c}}$

$T_{\mathrm{pr}}=\frac{T}{T_{c}}$,

$Z=\left(0.702 \mathrm{e}^{-2.5 T_{\mathrm{pr}}}\right) P_{\mathrm{pr}}^{2}-5.524 \mathrm{e}^{-2.5 T_{\mathrm{pr}}} P_{\mathrm{pr}}$ $+0.044 T_{\mathrm{pr}}^{2}-0.164 T_{\mathrm{pr}}+1.15$.

黏度计算采用Lee等人 ${ }^{[37]}$ 提出的方法. 该方法已被 其他学者应用到气体黏度计算中 ${ }^{[38,39]}$. Lee等人方法的 压力适用范围 0.69-55 MPa, 温度适用范围311-444.2 K. 本文中选用气藏压力为 $40 \mathrm{MPa}$, 地层温度为 $400 \mathrm{~K}$, 因 此符合适用条件.

$\mu=\left(1 \times 10^{-4}\right) K \exp \left(X \rho^{Y}\right)$,

$\rho=1.4935 \times 10^{-3} \frac{p_{o} M}{Z T}$,

$K=\frac{(9.379+0.01607 M) T^{1.5}}{(209.2+19.26 M+T)}$,

$X=3.448+\frac{986.4}{T}+0.01009 M$,

$Y=2.447-0.2224 X$.

\section{3 自由气传导率方程}

真实气体平均分子自由程可表示为 ${ }^{[40]}$

$\lambda=\sqrt{\frac{\pi Z R T}{2 M_{w}}} \frac{\mu}{p_{o}}$.

Knudsen数在有机质孔隙和无机质孔隙中可分别 表示为

$K_{n}=\frac{\lambda}{r_{\text {eff_or }}}$,

$K_{n}=\frac{\lambda}{r}$.

Beskok等人 ${ }^{[6]}$ 建立了基于Knudsen数的单管气体 流量公式, 如下式所示:

$q_{h}=f\left(K_{n}\right) \frac{\pi R_{h}^{4}}{8 \mu} \frac{\Delta P}{L_{h}}$,

其中, $f\left(K_{n}\right)$ 可表示为

$f\left(K_{n}\right)=\left(1+\alpha K_{n}\right)\left(1+\frac{4 K_{n}}{1-\beta K_{n}}\right)$.

无因次气体稀薄系数 $\alpha$ 可表示为

$\alpha=\frac{128}{15 \pi^{2}} \tan ^{-1}\left[4.0 K_{n}^{0.4}\right]$.

虽然滑移系数 $\beta$ 值为 -1 , 最初仅被使用于滑脱 流动阶段, Beskok与分子模拟数据对比显示滑移系 数 $\beta$ 值为 -1 可适用于全部Knudsen数流动阶段 ${ }^{[41]}$. 根 据式(16)自由气传导率可表示为

$g_{\text {free }}=\frac{\pi r_{\text {eff }}^{4} f\left(K_{n}\right)}{8 \mu L}$.

\section{4 吸附气传导率方程}

吸附气通过表面扩散方式进行流动. 单位面积气 体分子摩尔流量可表示为 ${ }^{[42,43]}$

$J_{a}=D_{s} \frac{\mathrm{d} C_{a}}{\mathrm{~d} x}$,

吸附气浓度 $C_{a}$ 通过Langmuir等温吸附计算 ${ }^{[30]}$ :

$C_{a}=C_{a \max } \theta$.

对于有机质孔隙, 不同页岩岩石干酪根类型不同, 气体吸附能力不同 ${ }^{[4,45]}$. 在本模型中, 孔隙材质对气体 
流动的影响是通过在模型中通过最大吸附浓度作为 模型参数进行输入来考虑. 有机质表面最大吸附浓度 $C_{a \max }$ 如下式所示 ${ }^{[46]}$ :

$C_{a \max }=\frac{V_{L} \rho_{\mathrm{sc}, \mathrm{gas}} \rho_{\text {grain }}}{\varepsilon_{k s} M}$.

根据式(20)-(22), 吸附层气体分子摩尔流量如下 式所示:

$J_{A}=D_{s} C_{a \max } \frac{\mathrm{d} \theta}{\mathrm{d} p} \pi\left(r^{2}-r_{\text {eff_or }}^{2}\right) \frac{\mathrm{d} p}{\mathrm{~d} x}$.

根据式(23), 吸附气体积流量可表示为

$V_{A}=\frac{M}{\rho} D_{s} C_{a \max } \frac{\mathrm{d} \theta}{\mathrm{d} p} \pi\left(r^{2}-r_{\text {eff_or }}^{2}\right) \frac{\mathrm{d} p}{\mathrm{~d} x}$.

考虑气体覆盖度的影响, 表面扩散系数 $D_{s}$ 采用如 下公式进行计算 ${ }^{[43,47,48]}$ :

$D_{s}=$

$D_{s 0} \frac{(1-\theta)+\frac{\kappa}{2} \theta(2-\theta)+\{H(1-\kappa)\}(1-\kappa) \frac{\kappa}{2} \theta^{2}}{\left(1-\theta+\frac{\kappa}{2} \theta\right)^{2}}$,

$D_{s 0}=8.29 \times 10^{-7} T^{0.5} \exp \left(-\frac{\Delta H^{0.8}}{R T}\right)$,

$H(1-\kappa)=\left\{\begin{array}{l}0, \kappa \geq 1 \\ 1,0 \leq \kappa \leq 1\end{array}\right.$

$\kappa=\frac{\kappa_{b}}{\kappa_{m}}$.

当 $\kappa_{m}>\kappa_{b}$, 不论运动前方的位置是否已被分子占据, 都将发生表面扩散; 当 $\kappa_{b}>\kappa_{m}$, 气体分子被堵塞, 表面扩 散停止.

根据式(24)吸附气传导率方程可表示为

$g_{\text {surface }}=\frac{1}{l} \frac{M}{\rho} D_{s} C_{a \max } \frac{\mathrm{d} \theta}{\mathrm{d} p} \pi\left(r^{2}-r_{\text {eff_or }}^{2}\right)$.

\section{5 有机质孔隙与无机质孔隙气体传导率方程}

有机质孔隙中气体以吸附气和自由气两种形式 流动, 根据式(19)和(29), 有机质孔隙气体传导率方程 可表示为

$$
\begin{aligned}
g_{\text {or }}= & \frac{\pi r_{\text {effor }}^{4}}{8 \mu L}\left(1+\frac{128}{15 \pi^{2}} \tan ^{-1}\left[4.0 K_{n}^{0.4}\right] K_{n}\right)\left(1+\frac{4 K_{n}}{1+K_{n}}\right) \\
& +\frac{1}{l} \frac{M}{\rho} D_{s} C_{a \max } \frac{\mathrm{d} \theta}{\mathrm{d} p} \pi\left(r^{2}-r_{\text {eff_or }}^{2}\right) .
\end{aligned}
$$

无机质孔隙中考虑自由气流动, 根据式(19)无机 质孔隙气体传导率方程可表示为

$$
\begin{aligned}
g_{\text {in }}= & \frac{\pi r^{4}}{8 \mu L}\left(1+\frac{128}{15 \pi^{2}} \tan ^{-1}\left[4.0 K_{n}{ }^{0.4}\right] K_{n}\right) \\
& \times\left(1+\frac{4 K_{n}}{1+K_{n}}\right) .
\end{aligned}
$$

\section{2 有机质孔隙与无机质孔隙单管流动能力 分析}

选取孔隙半径 $5,20,40 \mathrm{~nm}$ 单管在两端定压差情 况下进行气体流量计算, 由于气体传导率与压力有关, 采用非线性迭代求解, 模型参数见表 $1^{[43,46]}$.

从图 4 中可看出, 在 $r=5 \mathrm{~nm}$ 时有机质孔隙单管气体 流动能力要高于无机质孔隙单管流动能力, 这是由于 $r=5 \mathrm{~nm}$ 时有机质孔隙中吸附气表面扩散作用较强, 从 而提高了有机质孔隙气体流动能力. 当 $r=20,40 \mathrm{~nm}$ 时 (图5和6)吸附气表面扩散能力减弱, 但吸附层仍占据 孔隙表面一定厚度, 使自由气流动截面相比无机质孔 隙中自由气流动截面减少, 从而使有机质孔隙气体流

表 1 单管流动能力分析模型参数

Table 1 Single tube gas flow ability analysis model parameter

\begin{tabular}{cc}
\hline 模型参数 & 数值 \\
\hline 单管长度 $L(\mathrm{~nm})$ & 100 \\
孔隙压力 $p_{o}(\mathrm{MPa})$ & 40 \\
地层温度 $T(\mathrm{~K})$ & 400 \\
孔隙半径 $r(\mathrm{~nm})$ & $5,20,40$ \\
压力梯度 $P_{g}(\mathrm{MPa} / \mathrm{m})$ & $0.05,0.1,0.15,0.2,0.25$ \\
Langmuir压力 $P_{\mathrm{L}}(\mathrm{MPa})$ & $13.789514^{[46]}$ \\
最大吸附浓度 $C_{a \max }\left(\mathrm{mol} / \mathrm{m}^{3}\right)$ & $328.7^{[46]}$ \\
有机质占总岩石骨架体 & $0.01^{[46]}$ \\
积比例 $\varepsilon_{k s}$ & $2.66 \times 10^{3}$ \\
岩石密度 $\rho_{\text {grain }}\left(\mathrm{kg} / \mathrm{m}^{3}\right)$ & $16000^{[43]}$ \\
覆盖度 “ 0 ”时的等量吸附 & 0.5 \\
热 $\Delta H\left(\mathrm{~J} \mathrm{~mol}{ }^{-1}\right)$ & \\
气体壁面扩散阻塞系数 $\kappa^{[43]}$ & \\
\hline
\end{tabular}




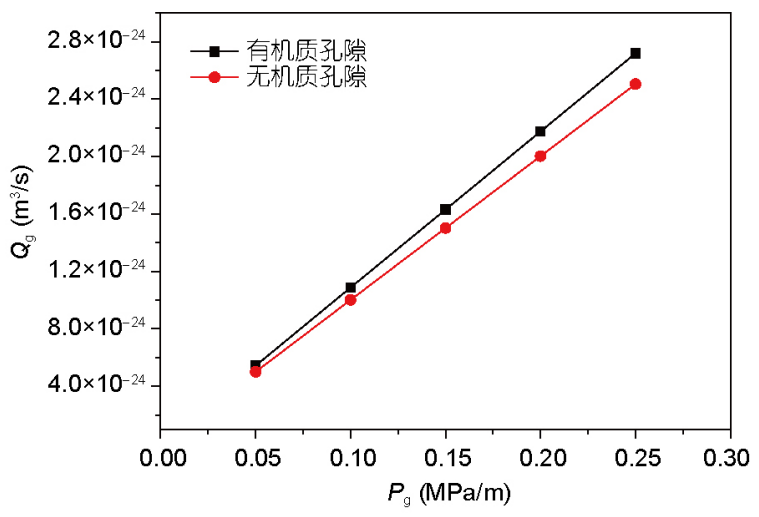

图 4 (网络版彩图) $r=5 \mathrm{~nm}$ 时两种孔隙类型单管气体流量 Figure 4 (Color online) Gas flux in two different types of tube when $r=5 \mathrm{~nm}$.

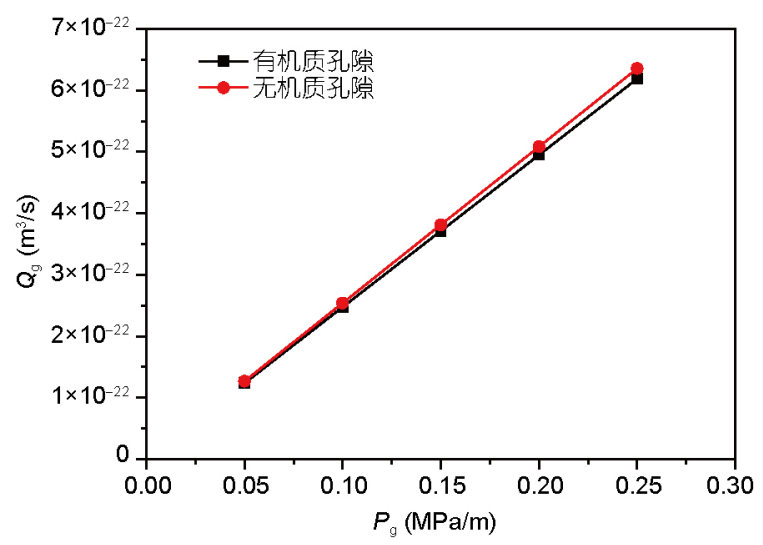

图 5 (网络版彩图) $r=20 \mathrm{~nm}$ 时有机质单管和无机质单管气 体流量

Figure 5 (Color online) Gas flux in two different types of tube when $r=20 \mathrm{~nm}$.

动能力小于无机质孔隙中气体流动能力. 单管流动能 力分析得到了有机质孔隙与无机质孔隙气体传输能 力的不同, 在第3节将单管流动规律应用到孔隙网络 模型中.

\section{3 页岩气孔隙网络模型}

\section{1 考虑有机质孔隙分布因素的页岩孔隙网络 模型}

图7所示某页岩剖光扫描电镜图像, 分辨率为 $12 \mathrm{~nm}$, 经过图像二值化处理后采用MCMC方法数值 重建三维页岩数字岩心 ${ }^{[49,50]}$. 重建后页岩数字岩心大 小为 $400 \times 400 \times 400$ (图8), 采用居中轴线法提取孔隙几

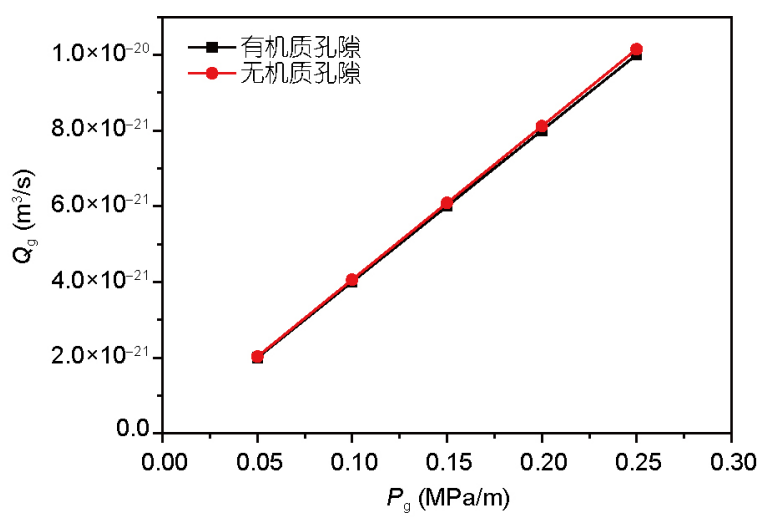

图 6 (网络版彩图) $r=40 \mathrm{~nm}$ 时有机质单管和无机质单管气 体流量

Figure 6 (Color online) Gas flux in two different types of tube when $r=40 \mathrm{~nm}$.

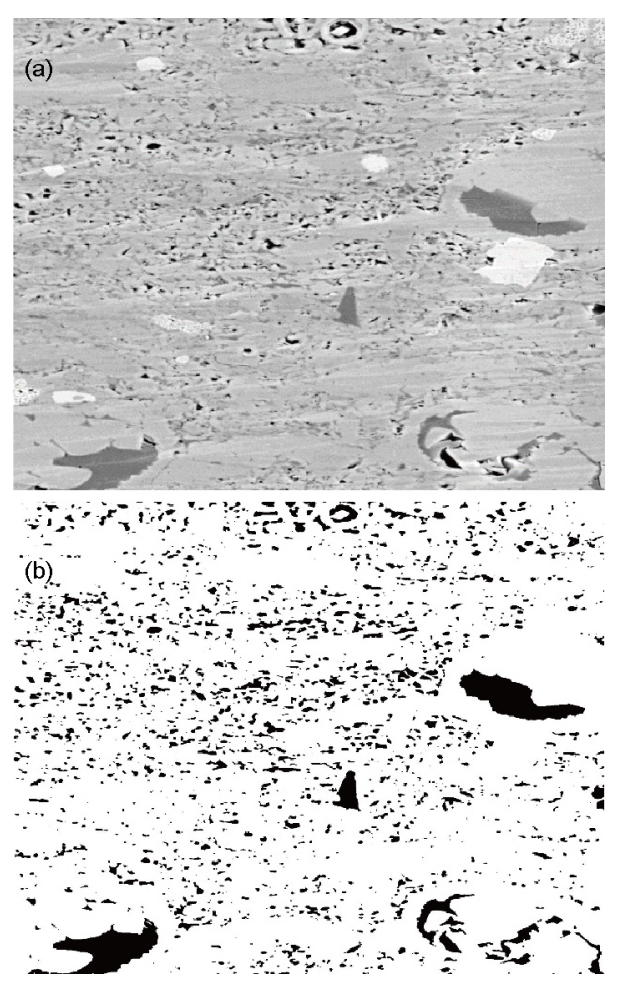

图 7 页岩二维剖光扫描电镜图像. (a)二值化前; (b) 二值 化后

Figure 7 Two dimensional polished shale SEM image. (a) Unsegmented image; (b) segmented image.

何拓扑 ${ }^{[51,52]}$, 提取后孔隙几何拓扑(图9)中孔隙个数为 49407 , 喉道个数为 79450 , 孤立孔隙个数为 32 . 有机质 孔隙基本标记单元为单个喉道和与之相连的两个孔 隙(图10). 在孔隙几何拓扑中标记孔隙类型, 建立3种 


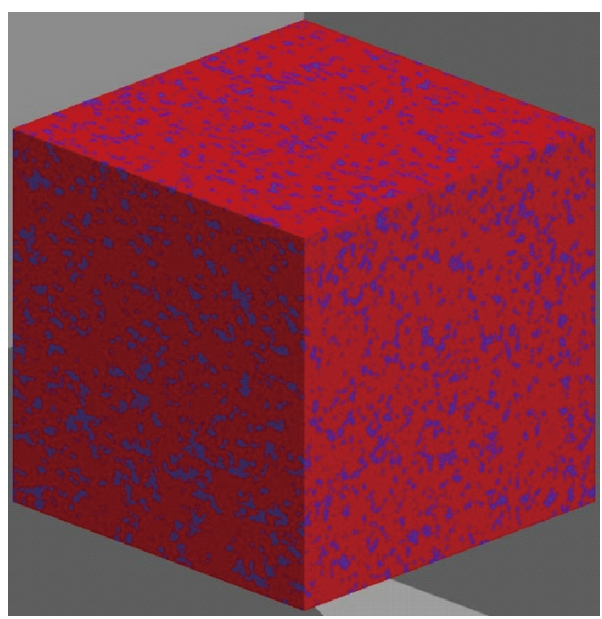

图 8 (网络版彩图) MCMC重建得到的三维页岩数字岩心 Figure 8 (Color online) Reconstructed three dimensional shale digital core based on MCMC algorithm.

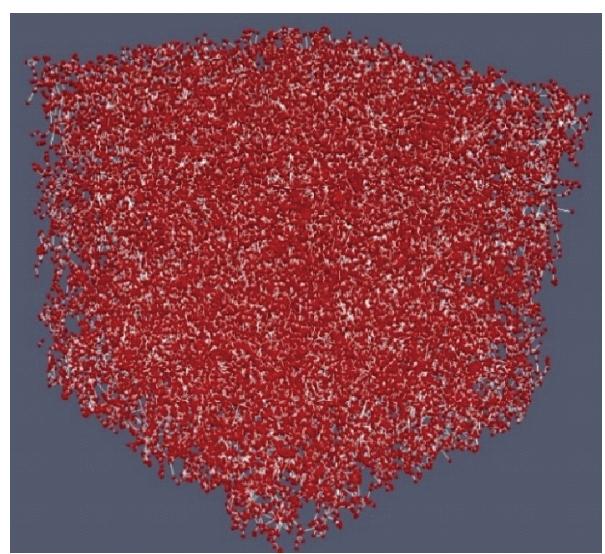

图 9 (网络版彩图)三维页岩数字岩心孔隙相中轴拓扑结 构(红色表示孔隙位置, 白色表示喉道位置)

Figure 9 (Color online) Medial axis in the pore space of three dimensional shale digital core (red color represents pore location, white color represents throat location).

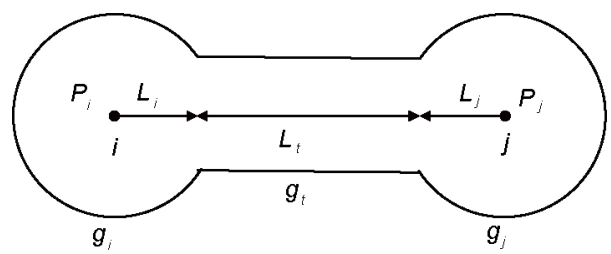

图 10 有机质孔隙基本标记单元(单个喉道和与之相连的 两个孔隙)

Figure 10 Basic labeling unit for organic pores (single throat with two connected pores).
页岩孔隙网络模型(图11-13). 3种页岩孔隙网络模型 中红色表示有机质孔隙,蓝色表示无机质孔隙.

3 种页岩孔隙网络模型中, 有机质孔隙数量占据 总孔隙数量均为 $10 \%$. 图 11 为 $\mathrm{A}$ 模式页岩孔隙网络模 型, 在孔隙网络上部和下部呈条带状分布, 中部为无 机质孔隙. 图12为B模式页岩孔隙网络模型, 有机质孔 隙在孔隙网络中连片分布, 有机质孔隙一部分位于孔 隙网络上部和底部, 并在孔隙网络中间位置从上至下 贯穿分布. 图13为 $C$ 模式页岩孔隙网络模型, 有机质孔 隙在孔隙网络中分散分布.

\section{2 有机质孔隙和无机质孔隙的半径分布}

页岩中有机质孔隙、无机质孔隙半径分布通常 呈现以 10 为底的对数正态分布 ${ }^{[33-55]}$, 表示形式如下:

$N(r$, ave,$\sigma)=\mathrm{e}^{\left.-\frac{\left(\log _{10^{r}-\text { ave }}\right)^{2}}{2 \sigma^{2}}\right] .}$

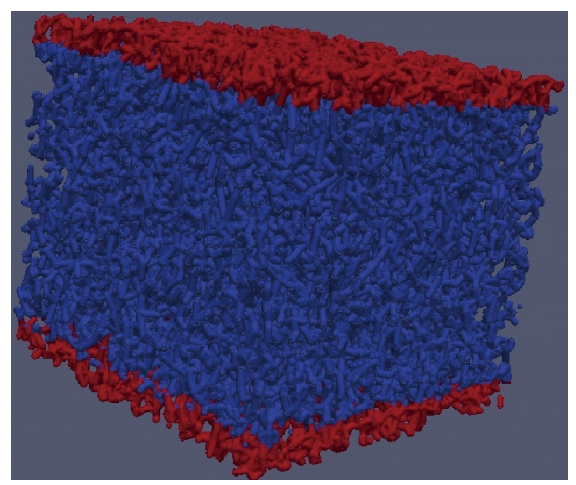

图 11 (网络版彩图) A模式页岩孔隙网络模型

Figure 11 (Color online) Pattern A shale pore network model.

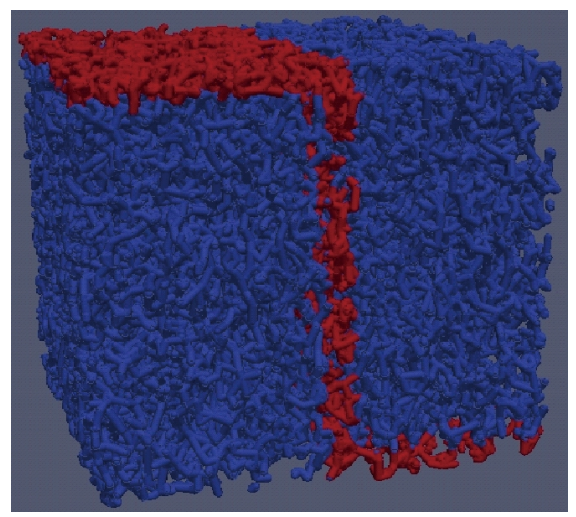

图 12 (网络版彩图) B模式页岩孔隙网络模型

Figure 12 (Color online) Pattern B shale pore network model. 


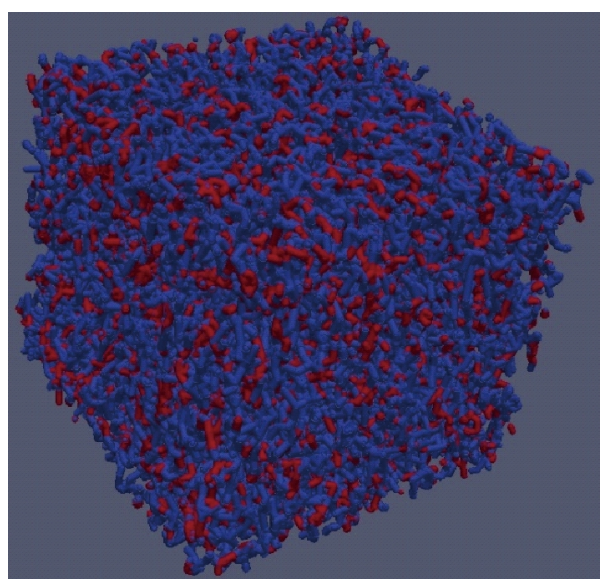

图 13 (网络版彩图) C模式页岩孔隙网络模型

Figure 13 (Color online) Pattern C shale pore network model.

根据国内某盆地页岩区块孔喉分析数据, 无机质 孔隙半径分布以及两组来自不同地层的有机质孔隙 半径分布参数见表2. 有机质孔隙和无机质孔隙半径 分布见图14. 从图14中可以看出, 有机质孔隙半径分 布 $a$ 小于无机质孔隙半径分布, 有机质孔隙半径分布 $b$ 大于无机质孔隙半径分布. 在 3 种页岩孔隙网络模型 中, 无机质孔隙半径大小按照无机质孔隙半径分布设 置, 有机质孔隙半径大小分别按照 $a b$ 两种不同有机质 孔隙半径分布设置, 产生6组不同孔隙分布模式、不 同孔径分布的页岩孔隙网络模型. 根据式(13)-(15)计 算得到的Knudsen数在有机质孔隙以及无机质孔隙中 随孔隙半径变化见图15. 由于吸附层的存在, 有机质 孔隙Knudsen数稍大于无机质孔隙Knudsen数. 孔隙半 径在 $10 \mathrm{~nm}$ 以下时自由气流动主要处于滑移流动和过 渡流动区域, $10 \mathrm{~nm}$ 以上时自由气流动高压时主要处 于连续流动区域, 低压时主要处于滑移流动区域.

\section{3 页岩气孔隙网络流动模拟}

对于每个孔隙气体流入流出量相等可表示为

$\sum_{j=1}^{N_{i}} Q_{i j}=0$,

$Q_{i j}=g_{i j}\left(p_{i}-p_{j}\right)$.

根据图 10, 相连孔隙间传导率可表示为 ${ }^{[56]}$

$\frac{L_{i}+L_{t}+L_{j}}{g_{i, j}}=\frac{L_{i}}{g_{i}}+\frac{L_{t}}{g_{t}}+\frac{L_{j}}{g_{j}}$.

对孔隙网络整体非线性迭代求解得到定压差条件
表 2 有机质无机质孔隙半径分布模型参数

Table 2 Organic pore and inorganic pore size distribution model parameters

\begin{tabular}{ccc}
\hline 孔隙类型 & 均值ave & 标准差 $\sigma$ \\
\hline 有机质孔隙半径分布 $a$ & 0.7 & 0.12 \\
有机质孔隙半径分布 $b$ & 1.7 & 0.12 \\
无机质孔隙半径分布 & 1.2 & 0.14 \\
\hline
\end{tabular}

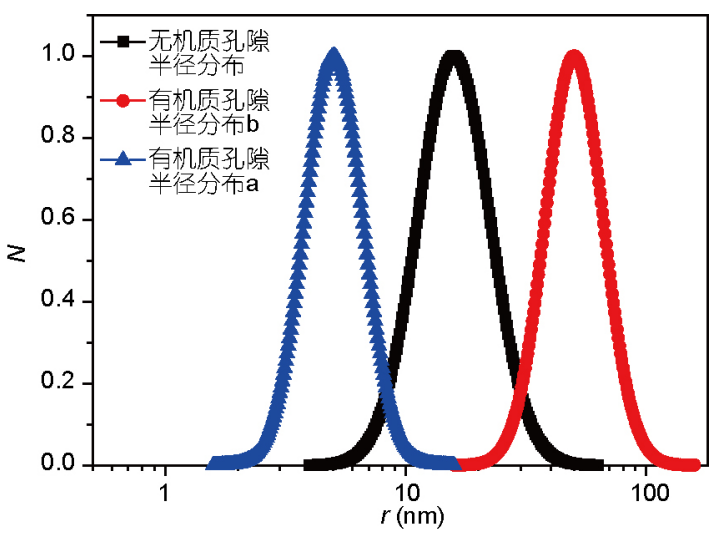

图 14 (网络版彩图)有机质无机质孔隙半径分布

Figure 14 (Color online) Organic pores and inorganic pores size distributions.

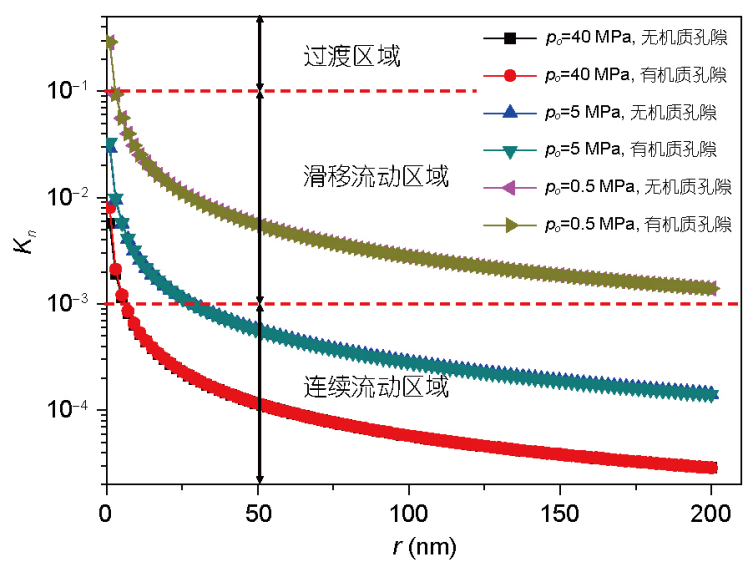

图 15 (网络版彩图) Knudsen数随孔隙半径以及孔隙压力 的变化

Figure 15 (Color online) Knudsen number change versus pore radius and pore pressure.

下通过孔隙网络模型的气体流量 $Q_{t}$, 初始参数见表 1 . 图16为压力梯度 $0.1 \mathrm{MPa} / \mathrm{m}$ 两端压差 $0.48 \mathrm{~Pa}$ 情况下, 6 组不同孔隙分布模式、不同孔径分布的页岩孔隙网 络模型压力分布计算结果, 为减少可视化内存需要, 喉道半径不予显示. 

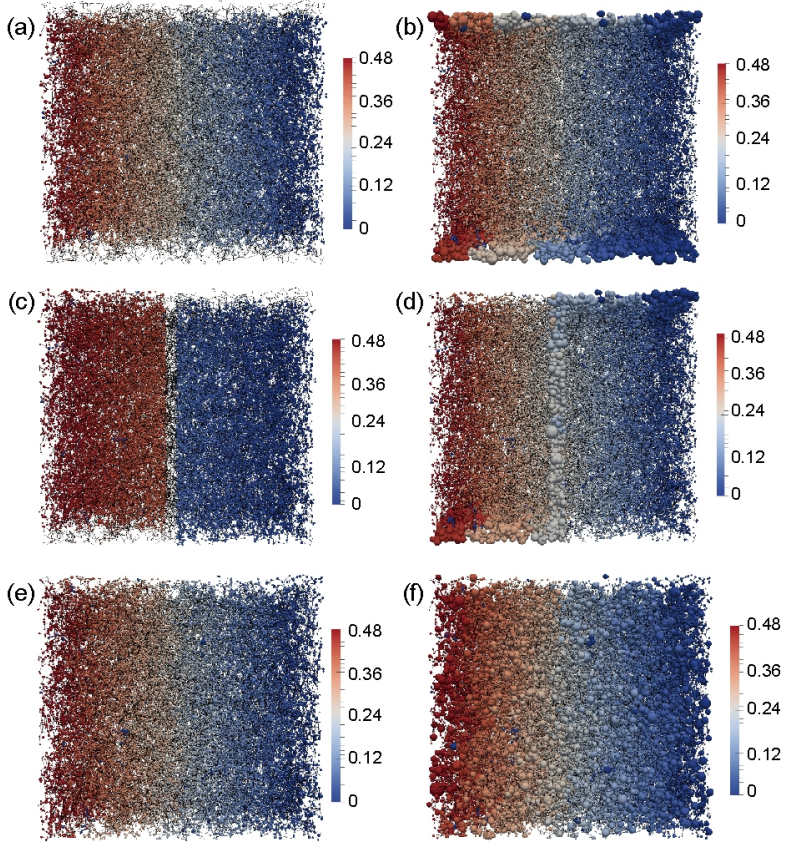

图 16 (网络版彩图) 6组不同孔隙分布模式不同孔径分布 的页岩孔隙网络模型压力分布计算结果. (a) A 模式-有机质 孔径分布 $a$; (b) A 模式-有机质孔径分布 $b$; (c) B 模式-有机质 孔径分布 $a$; (d) B模式-有机质孔径分布 $b$; (e) C模式-有机质 孔径分布 $a$; (f) C模式-有机质孔径分布 $b$

Figure 16 (Color online) Shale pore network pressure distribution calculation results under different pore size distribution and different organic pore distribution pattern. (a) Pattern A-organic pore size distribution $a$; (b) Pattern A-organic pore size distribution $b$; (c) Pattern B-organic pore size distribution $a$; (d) Pattern B-organic pore size distribution $b$; (e) Pattern $\mathrm{C}$-organic pore size distribution $a$; (f) Pattern C-organic pore size distribution $b$.

图17为有机质孔隙半径分布小于无机质孔隙半 径分布时, 3 种页岩孔隙网络模型气体流量随压力梯 度的变化情况. 有机质孔隙成条带状分布的A模式页 岩孔隙网络模型气体流动能力最强, 有机质孔隙分散 在无机质孔隙中的 $\mathrm{C}$ 模式页岩孔隙网络模型气体流动 能力次之, 有机质孔隙连片分布的B模式页岩孔隙网 络模型气体流动能力最弱. 这是由于有机质孔隙连片 分布时, 在孔隙网络模型中央形成相对于无机质孔隙 传导率低的屏障, 导致气体流动能力降低, 如图 16(c) 所示, 气体通过有机质孔隙需要克服更大的流动阻力, 因此在有机质孔隙上压力降较大. 有机质孔隙成条带 状分布时, 气体主要在中部无机质孔隙流动, 形成高 流动能力的通道, 因此相比其他两种孔隙网络模型, 气体流动能力最强.

图18为有机质孔隙半径分布大于无机质孔隙半径

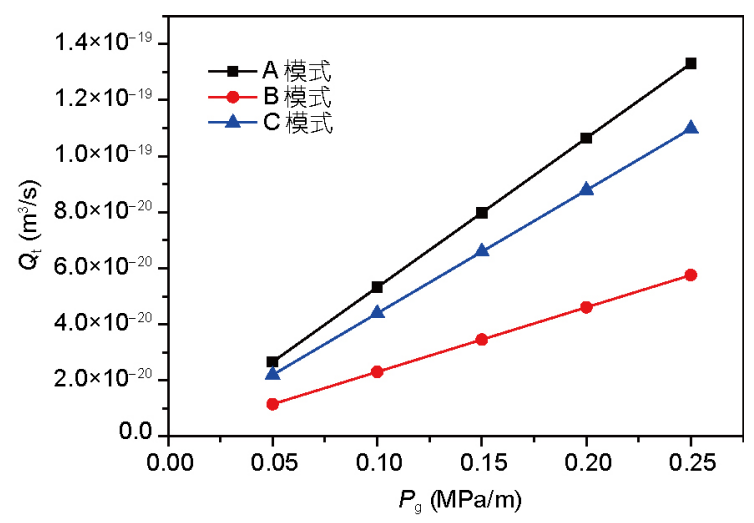

图 17 (网络版彩图)有机质孔径分布 $a$ 下 3 种页岩孔隙网络 模型气体流量随压力梯度变化

Figure 17 (Color online) Gas flux versus pressure gradient in three different types of shale pore network under the organic pore size distribution

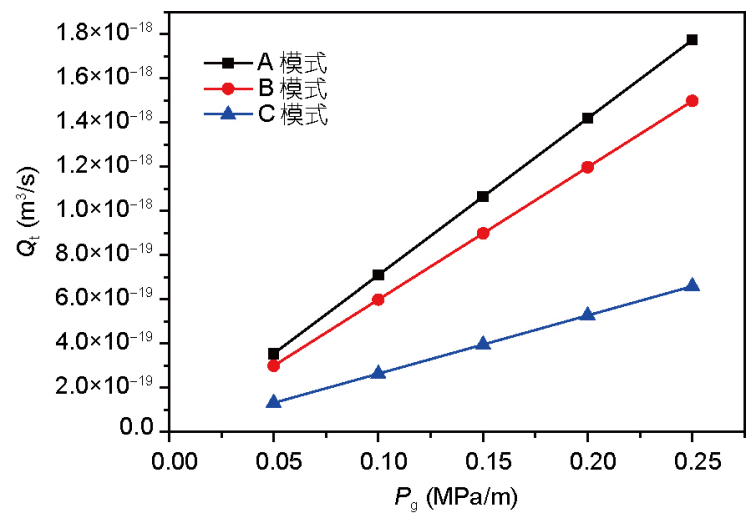

图 18 有机质孔径分布 $b$ 下 3 种页岩孔隙网络模型气体流 量随压力梯度变化

Figure 18 Gas flux versus pressure gradient in three different types of shale pore network under the organic pore size distribution $b$.

分布时, 3 种页岩孔隙网络模型气体流量随压力梯度 的变化情况. 有机质孔隙成条带状分布的 $\mathrm{A}$ 模式页岩 孔隙网络模型气体流动能力最强, 有机质孔隙连片分 布的 $\mathrm{B}$ 模式页岩孔隙网络模型气体流动能力次之, 有 机质孔隙分散在无机质孔隙中的 $\mathrm{C}$ 模式页岩孔隙网络 模型气体流动能力最弱. 这是由于 $\mathrm{C}$ 模式页岩孔隙网 络模型气体流动能力主要由孔径较小的无机质孔隙 控制, 孔隙半径分布较大的有机质孔隙分散在无机质 孔隙中, 因此 $\mathrm{C}$ 模式页岩孔隙网络模型气体流动能力 变化幅度较小. A 模式页岩孔隙网络模型顶部和底部 形成高渗条带, 气体流动能力有很大提高. $\mathrm{B}$ 模式页岩 孔隙网络模型形成 $Z$ 型高渗条带, 因此气体流动能力 
也有提高, 但由于相对于 $\mathrm{A}$ 模式, 高渗区域曲折分布, 因此气体流量小于 $\mathrm{A}$ 模式下的气体流量.

\section{4 结论}

根据地质上对有机质孔隙分布、有机质孔隙无 机质孔隙半径分布的认识, 建立了3种页岩孔隙网络 模型: 有机质孔隙成条带状分布的 $\mathrm{A}$ 模式页岩孔隙网 络模型、有机质孔隙连片分布的B模式页岩孔隙网络 模型、有机质孔隙分散在无机质孔隙中的 $\mathrm{C}$ 模式页岩 孔隙网络模型. 有机质孔隙中气体流动考虑了吸附解 吸、表面扩散、黏性流、努森扩散, 无机质孔隙中气 体流动考虑了黏性流、努森扩散, 气体性质计算考虑 了真实气体效应以及气体在微纳米孔道产生的相态 变化.

当有机质孔隙半径分布小于无机质孔隙半径分 布时, 有机质孔隙成条带状分布的 $\mathrm{A}$ 模式页岩孔隙网
络模型气体流动能力最强, 有机质孔隙分散在无机质 孔隙中的 $\mathrm{C}$ 模式页岩孔隙网络模型气体流动能力次之, 有机质孔隙连片分布的B模式页岩孔隙网络模型气体 流动能力最弱.

当有机质孔隙半径分布大于无机质孔隙半径分 布时, 有机质孔隙成条带状分布的 $\mathrm{A}$ 模式页岩孔隙网 络模型气体流动能力最强, 有机质孔隙连片分布的 $\mathrm{B}$ 模式页岩孔隙网络模型气体流动能力次之, 有机质孔 隙分散在无机质孔隙中的 $\mathrm{C}$ 模式页岩孔隙网络模型气 体流动能力最弱.

本文建立的页岩孔隙网络模型考虑了页岩地层 中有机质孔隙无机质孔隙的地质分布模式, 并且考虑 了有机质孔隙与无机质孔隙在气体传输、孔径分布 上的差异. 相比于传统页岩气单管表观渗透率模型, 本文提出的页岩孔隙网络模型更能准确地预测页岩 气流动能力.

\section{参考文献}

1 Yao J, Sun H, Huang Z Q, et al. Key mechanical problems in the developmentof shale gas reservoirs (in Chinese). Sci Sin-Phys Mech Astron, 2013, 43: 1527-1547 [姚军, 孙海, 黄朝琴, 等. 页岩气藏开发中的关键力学问题. 中国科学: 物理学 力学 天文学, 2013, 43: 1527-1547]

2 Yao J, Sun H, Fan D, et al. Numerical simulation of gas transport mechanisms in tight shale gas reservoirs. Pet Sci, 2013, 10: 528-537

3 Sun H, Yao J, Fan D, et al. Gas transport mode criteria in ultra-tight porous media. Int J Heat Mass Transf, 2015, 83: 192-199

4 Song W, Yao J, Li Y, et al. Apparent gas permeability in an organic-rich shale reservoir. Fuel, 2016, 181: 973-984

5 Javadpour F, Fisher D, Unsworth M. Nanoscale gas flow in shale gas sediments. J Canadian Pet Tech, 2007, 46: 56-61

6 Beskok A, Karniadakis G H. Report: A model for flows in channels, pipes, and ducts at micro and nano scales. Microscale Thermophys Eng, 1999, 3: 43-77

7 Civan F. Effective correlation of apparent gas permeability in tight porous media. Transp Porous Med, 2010, 82: 375-384

8 Civan F, Rai C S, Sondergeld C H. Shale-gas permeability and diffusivity inferred by improved formulation of relevant retention and transport mechanisms. Transp Porous Med, 2011, 86: 925-944

9 Xiong X, Devegowda D, Villazon M, et al. A fully-coupled free and adsorptive phase transport model for shale gas reservoirs including non-darcy flow effects. In: Proceedings of SPE Annual Technical Conference and Exhibition. San Antonio, 2012

10 Javadpour F. Nanopores and apparent permeability of gas flow in mudrocks (shales and siltstone). J Canadian Pet Tech, 2009, 48: 16-21

11 Singh H. Nonempirical apparent permeability of shale. In: Proceedings of Unconventinal Resources Technology Conference. Denver: AAPG, 2013

12 Singh H, Javadpour F. Langmuir slip-Langmuir sorption permeability model of shale. Fuel, 2016, 164: 28-37

13 Wu K, Chen Z, Li X, et al. A model for multiple transport mechanisms through nanopores of shale gas reservoirs with real gas effect-adsorptionmechanic coupling. Int J Heat Mass Transf, 2016, 93: 408-426

14 Wu K, Chen Z, Wang H, et al. A model for real gas transfer in nanopores of shale gas reservoirs. In: Proceedings of Unconventinal Resources Technology Conference. Madrid: AAPG, 2013

15 Wu K L, Li X F, Chen Z X. A model for gas transport through nanopores of shale gas reservoirs (in Chinese). Acta Pet Sin, 2015, 36: 837-848 [吴克柳, 李相方, 陈掌星. 页岩气纳米孔气体传输模型. 石油学报, 2015, 36: 837-848]

16 Fishman N S, Hackley P C, Lowers H A, et al. The nature of porosity in organic-rich mudstones of the Upper Jurassic Kimmeridge clay formation, North Sea, offshore United Kingdom. Int J CoalGeol, 2012, 103: 32-50

17 Song W, Yao J, Li Y, et al. New pore size distribution calculation model based on chord length and digital image. J Nat Gas Sci Eng, 2016, doi: 
10.1016/j.jngse.2016.12.041

18 Mastalerz M, Schimmelmann A, Drobniak A, et al. Porosity of devonian and Mississippian new Albany shale across a maturation gradient: Insights from organic petrology, gas adsorption, and mercury intrusion. Bulletin, 2013, 97: 1621-1643

19 Rine J, Dorsey W, Floyd M, et al. A comparative SEM study of pore types and porosity distribution in high to low porosity samples from selected gas-shale formations. Gulf Coast Associat Geol Soc Trans, 2010, 60: 825

20 Loucks R G, Reed R M, Ruppel S C, et al. Morphology, genesis, and distribution of nanometer-scale pores in siliceous mudstones of the mississippian barnett shale. J Sediment Res, 2009, 79: 848-861

21 Li X F, Pu Y C, Sun C Y, et al. Recognition of adsorption/desorption theory in coalbed methane reservoir and shale gas reservoir (in Chinese). Acta Pet Sin, 2014, 35: 1113-1129 [李相方, 蒲云超, 孙长宇. 煤层气与页岩气吸附/解吸的理论再认识. 石油学报, 2014, 35: 1113-1129]

22 Li J, Li X F, Wang X Z, et al. Effect of water distribution on methane adsorption capacity in shale clay (in Chinese). Chin J Theoretical Appl Mech, 2016, 48: 1217-1228 [李靖, 李相方, 王香增. 页岩黏土孔隙含水饱和度分布及其对甲烷吸附的影响. 力学学报, 2016, 48: 1217-1228]

23 Zhang X F, Lu X C, Zhang L Y, et al. Occurrences of shale gas and their petroleum geological significance (in Chinese). Adv Earth Sci, 2010, 25: 597-604 [张雪芬, 陆现彩, 张林晔, 等. 页岩气的赋存形式研究及其石油地质意义. 地球科学进展, 2010, 25: 597-604]

24 Wang P Q, Li X H. Thermal weightlessness method to determine water content and existing form of hydratable clay (in Chinese). Nat Gas Ind, 2006, 26: 80-83 [王平全, 李晓红. 用热失重法确定水合黏土水分含量及存在形式. 天然气工业, 2006, 26: 80-83]

25 Odusina E O, Sondergeld C H, Rai C S. NMR study of shale wettability. In: Proceedings of Canadian Unconventional Resources Conference. Calgary: Society of Petroleum Engineers, 2011

26 Zhao Z G. Principle of Adsorption Application (in Chinese). Beijing: Chemical Industry Press, 2005 [赵振国. 吸附作用应用原理. 北京: 化 学工业出版社, 2005

27 Jin Z, Firoozabadi A. Effect of water on methane and carbon dioxide sorption in clay minerals by Monte Carlo simulations. Fluid Phase Equilib, 2014, 382: 10-20

28 Teng X R. Surface Physical Chemistry (in Chinese). Beijing: Chemical Industry Press, 2009 [滕新荣. 表面物理化学. 北京: 化学工业出版 社, 2009]

29 Wang Y, Zhu Y, Liu S, et al. Pore characterization and its impact on methane adsorption capacity for organic-rich marine shales. Fuel, 2016, 181: $227-237$

30 Heller R, Zoback M. Adsorption of methane and carbon dioxide on gas shale and pure mineral samples. J Unconven Oil Gas Resour, 2014, 8: $14-24$

31 Kang S M, Fathi E, Ambrose R J, et al. Carbon dioxide storage capacity of organic-rich shales. SPE J, 2011, 16: 842-855

32 Ambrose R J, Hartman R C, Diaz C M, et al. New pore-scale considerations for shale gas in place calculations. In: Proceedings of Canadian Unconventional Resources Conference. Calgary: Society of Petroleum Engineers, 2011

33 Islam A W, Patzek T W, Sun A Y. Thermodynamics phase changes of nanopore fluids. J Nat Gas Sci Eng, 2015, 25: 134-139

34 Mahmoud M. Development of a new correlation of gas compressibility factor (Z-factor) for high pressure gas reservoirs. J Energ Resour Technol, 2014, 136: 012903

35 Fan X, Li G, Shah S N, et al. Analysis of a fully coupled gas flow and deformation process in fractured shale gas reservoirs. J Nat Gas Sci Eng, 2015, 27: 901-913

36 Yu W, Sepehrnoori K, Patzek T W. Modeling gas adsorption in marcellus shale with Langmuir and BET isotherms. SPE J, 2016, 21: 589-600

37 Lee A L, Gonzalez M H, Eakin B E. The viscosity of natural gases. J Pet Tech, 1966, 18: 997-991

38 Landry C J, Prodanović M, Eichhubl P. Direct simulation of supercritical gas flow in complex nanoporous media and prediction of apparent permeability. Int J CoalGeol, 2016, 159: 120-134

39 Kim C, Jang H, Lee Y, et al. Diffusion characteristics of nanoscale gas flow in shale matrix from Haenam basin, Korea. Environ Earth Sci, 2016, 75: 350

40 Villazon M, German G, Sigal R F, et al. Parametric investigation of shale gas production considering nano-scale pore size distribution, formation factor, and non-darcy flow mechanisms. In: Proceedings of SPE Annual Technical Conference and Exhibition. Denver: Society of Petroleum Engineers, 2011

41 Karniadakis G E, Beskok A, Aluru N. Microflows and Nanoflows: Fundamentals and Simulation. New York: Springer Science \& Business Media, 2006

42 Cunningham R E, Williams R. Diffusion in Gases and Porous Media. Boston: Springer, 1980

43 Wu K, Li X, Wang C, et al. Model for surface diffusion of adsorbed gas in nanopores of shale gas reservoirs. Ind Eng Chem Res, 2015, 54: $3225-3236$ 
44 Yan J P, Zhang T W, Li Y F, et al. Effect of the organic matter characteristics on methane adsorption in shale (in Chinese). J China Coal Soc, 2013,38: 805-811 [闻建萍, 张同伟, 李艳芳. 页岩有机质特征对甲烷吸附的影响. 煤炭学报, 2013, 38: 805-811]

45 Cao T T, Song Z G. Effect of the organic matter characteristics on organic pore and reservoir (in Chinese). Spec Oil Gas Reserv, 2016, 4: 1-10 [曹涛涛, 宋之光. 页岩有机质特征对有机孔发育及储层的影响. 特种油气藏, 2016, 4: 1-10]

46 Wasaki A, Akkutlu I Y. Permeability of organic-rich shale. SPE J, 2015, 20: 1384-1396

47 Chen Y D, Yang R T. Concentration dependence of surface diffusion and zeolitic diffusion. AIChE J, 1991, 37: 1579-1582

48 Guo L, Peng X, Wu Z. Dynamical characteristics of methane adsorption on monolith nanometer activated carbon. J Chem Ind Eng, 2008, 59: 2726-2732

49 Yao J, Zhao X C, Yi Y J. The current situation and prospect on digital core technology (in Chinese). Pet Geol Recov Efficiency, 2005, 12: 52-54 [姚军, 赵秀才, 衣艳静. 数字岩心技术现状及展望. 油气地质与采收率, 2005, 12: 52-54]

50 Yang Y, Yao J, Wang C, et al. New pore space characterization method of shale matrix formation by considering organic and inorganic pores. J Nat Gas Sci Eng, 2015, 27: 496-503

51 Zhao X C. Numerical rock construction and pore network extraction (in Chinese). Dongying: China University of Petroleum (East), 2009 [赵秀 才. 数字岩心及孔隙网络模型重构方法研究. 东营: 中国石油大学 (华东), 2009]

52 Lee T C, Kashyap R L, Chu C N. Building skeleton models via 3-D medial surface axis thinning algorithms. CVGIP-Graphical Model Image Proc, 1994, 56: 462-478

53 Naraghi M E, Javadpour F. A stochastic permeability model for the shale-gas systems. Int J CoalGeol, 2015, 140: 111-124

54 Wang J, Chen L, Kang Q, et al. Apparent permeability prediction of organic shale with generalized lattice Boltzmann model considering surface diffusion effect. Fuel, 2016, 181: 478-490

55 Hinai A A, Rezaee R, Esteban L, et al. Comparisons of pore size distribution: A case from the Western Australian gas shale formations. J Unconven Oil Gas Resources, 2014, 8: 1-13

56 Valvatne P H. Predictive Pore-scale Modelling of Multiphase Flow. London: Imperial College London, 2004 


\title{
Study on the influence of organic pores on shale gas flow ability
}

\author{
YAO Jun ${ }^{1 *}$, SONG WenHui ${ }^{1}$, LI Yang ${ }^{2}$, SUN Hai ${ }^{1}$, YANG YongFei ${ }^{1} \&$ ZHANG Lei ${ }^{1}$ \\ ${ }^{1}$ School of Petroleum Engineering, China University of Petroleum (East), Qingdao 266580, China; \\ ${ }^{2}$ Department of Oilfield Exploration \& Development, China Petro-Chemical Corporation, Beijing 100029, China
}

In shale gas reservoirs, gas is stored in organic pores and inorganic pores with $\mathrm{nm}$-scale pore size. Conventional single tube apparent permeability model is based on homogeneity assumption and neglects the influences of organic pores spatial distribution pattern, pore size distribution on gas flow ability. In this study, three dimensional shale digital core is reconstructed by Markov Chain Monte Carlo (MCMC) algorithm and medial axis in the pore space is extracted. According to geological study on the organic pores distribution pattern and pore size distribution, different sizes of organic pores and inorganic pores are assigned to corresponding spatial location of pores and throats. Three different types of shale pore network are built: shale pore network model with dense continuous layers of organic pores; shale pore network model with sparse continuous layers of organic pores; shale pore network model with discontinuous organic pores. Gas transport mechanisms of adsorption and desorption, surface diffusion, viscous flow, Knudsen diffusion are considered in organic pores. Gas transport mechanisms of viscous flow, Knudsen diffusion are considered in inorganic pores. Gas property calculation takes the influences of real gas effects and phase behavior change into account. Analysis results indicate that when the organic pores size distribution is smaller than inorganic pores size distribution, the permeability of shale pore network model with dense continuous layers of organic pores is larger than that of the other two types of shale pore network model. The permeability of shale pore network model with sparse continuous layers of organic pores is smallest. When the organic pores size distribution is bigger than inorganic pores size distribution, the permeability of shale pore network model with dense continuous layers of organic pores is also larger than that of the other two types of shale pore network model. The permeability of shale pore network model with discontinuous organic pores is smallest. Our study results are significant for understanding gas flow ability in shale gas reservoirs.

shale gas, transport mechanisms, pore network model, organic pores, inorganic pores

PACS: $47.56 .+\mathrm{r}, 51.10 .+\mathrm{y}, 91.60 . \mathrm{Np}$

doi: $10.1360 /$ SSPMA2017-00040 